\title{
Genetic components of milk Fourier-transform infrared spectra used to predict breeding values for milk composition and quality traits in dairy goats
}

\author{
B. S. Dagnachew, ${ }^{1}$ T. H. E. Meuwissen, and T. Ådnøy \\ Department of Animal and Aquacultural Sciences, Norwegian University of Life Sciences, PO Box 5003, N-1432 Ås, Norway
}

\begin{abstract}
The usual practice today is that milk component phenotypes are predicted using Fourier-transform infrared (FTIR) spectra and they are then, together with pedigree information, used in BLUP for calculation of individual estimated breeding values. Here, this is referred to as the indirect prediction (IP) approach. An alternative approach - a direct prediction (DP) method - is proposed, where genetic analyses are directly conducted on the milk FTIR spectral variables. Breeding values of all derived milk traits (protein, fat, fatty acid composition, and coagulation properties, among others) can then be predicted as traits correlated only to the genetic information of the spectra. For the DP, no need exists to predict the phenotypes before calculating breeding values for each of the traits - the genetic analysis is done once for the spectra, and is applicable to all traits derived from the spectra. The aim of the study was to compare the effects of DP and IP of milk composition and quality traits on prediction error variance $(\mathrm{PEV})$ and genetic gain. A data set containing 27,927 milk FTIR spectral observations and milk composition phenotypes (fat, lactose, and protein) belonging to 14,869 goats of 271 herds was used for training and evaluating models. Partial least squares regression was used for calibrating prediction models for fat, protein, and lactose percentages. Restricted maximum likelihood was used to estimate variance components of the spectral variables after principal components analysis was applied to reduce the spectral dimension. Estimated breeding values were predicted for fat, lactose, and protein percentages using DP and IP methods. The DP approach reduced the mean PEV by $3.73,4.07$, and $7.04 \%$ for fat, lactose, and protein percentages, respectively, compared with the IP method. Given the reduction in PEV, relative genetic gains were $2.99,2.78$, and $4.85 \%$ for fat, lactose, and protein percentages, respectively. We concluded
\end{abstract}

Received August 18, 2012.

Accepted May 13, 2013.

${ }^{1}$ Corresponding author: binyam.dagnachew@umb.no that more accurate estimated breeding values could be found using genetic components of milk FTIR spectra compared with single-trait animal model analyses on phenotypes predicted from the spectra separately. The potential and application is not only limited to milk FTIR spectra, but could also be extended to any spectroscopy techniques implemented in other species and for other traits.

Key words: Fourier-transform infrared spectra, breeding value, prediction error variance, genetic gain

\section{INTRODUCTION}

In Europe, goat milk is mainly used for cheese production. Producing quality cheese requires quality raw milk - milk with good cheese-making ability. The quality of goat milk for cheese making is strongly related to the total amount and type of proteins, minerals, lipids, and coagulation properties (Grosclaude and Martin, 1997; Hallén, 2008). These properties of the milk are influenced by several genetic and environmental factors (Jaubert, 1996). Selection of dairy animals for improving the cheese-making ability of the milk is possible because genetic variation for most milk composition and quality traits exist (Ikonen, 2000; Manfredi, 2003; Cassandro et al., 2008; Schopen, et al., 2009; Cecchinato et al., 2011b).

Genetic evaluation of dairy animals requires rather large data sets, possibly larger than what is feasible to observe directly through chemical analyses for milk composition and quality traits because they are costly and time consuming. An alternative and more convenient method-use of infrared (IR) spectra-has been proposed (Goulden, 1964). Fourier-transform IR (FTIR) spectrometry is among several vibration spectroscopy techniques applied in food analysis, which use the mid-infrared region of the light spectrum (van de Voort, 1992). The FTIR spectra, exhibiting reduced cost of analysis, high throughput, and possibly large-scale application, are widely used today in milkrecording programs for milk payment and prediction of major milk components (e.g., protein, fat, and lactose percentages). 
The common practice today is that milk composition phenotypes are collected using FTIR spectra. These phenotypes are obtained through application of calibration equations on the spectral data. The predicted phenotypes are then, together with pedigree information and variance component estimates, used in BLUP for estimation of EBV, and selection candidates are ranked accordingly. In this paper, this approach is referred to as the indirect prediction (IP) method.

This study proposed an alternative approach - direct prediction (DP) method - where genetic analyses are directly conducted on the milk FTIR spectral variables. The BLUP breeding values for milk composition traits are then predicted as correlated traits to the genetic component of the spectra. The proposed method involves genetic analysis of several hundreds of spectral variables and is expected to benefit from use of information-rich spectral data. Studies have showed that milk FTIR spectral variables have strong correlations among each other in cow milk spectra (Soyeurt et al., 2010) and in goat milk spectra (Dagnachew and Ådnøy, 2011). Direct genetic analysis on these correlated spectral variables may provide a better estimation of genetic parameters and, hence, improve accuracy of animal evaluation methods.

Genetic evaluation of Norwegian dairy goats is based on major milk components predicted from FTIR spectra. These milk component measurements, together with pedigree information, are used for selection of superior individuals following the IP approach as explained above. The main aim of this study was to investigate the effect of using the DP approach for prediction of breeding values instead of the IP approach on accuracy and prediction error variance (PEV) for major milk components (i.e., fat, lactose, and protein percentages). We did not have independent chemical analyses of the milk components, but relied on predictions of milk content based on the same FTIR spectra as found in the Norwegian Dairy Goat Control.

\section{MATERIALS AND METHODS}

\section{FTIR Spectra and Phenotype Data}

In the Norwegian Dairy Goat Control, daily milk yield is to be recorded at least 5 times per farm per year. Of the 5 recording days, at least $3 \mathrm{~d}$ of individual goat samples should be analyzed for major milk components (fat percentage, protein percentage, lactose percentage, SCC, FFA, and urea content). The milk samples are analyzed by the MilkoScan CombiFoss 6500 instrument (Foss Electric A/S, Hillerød, Denmark) and the components are predicted using equations applied to the spectral data. The predictions are transferred
Table 1. Data structure: number of herds, goats, and individual test Fourier-transform infrared (FTIR) spectra

\begin{tabular}{|c|c|c|c|}
\hline Item & $\begin{array}{c}\text { Herds } \\
\text { (no.) }\end{array}$ & $\begin{array}{c}\text { Goats } \\
\text { (no.) }\end{array}$ & $\begin{array}{l}\text { Tests } \\
\text { (no.) }\end{array}$ \\
\hline \multicolumn{4}{|l|}{ Data set $^{1}$} \\
\hline \multicolumn{4}{|l|}{ Calibration } \\
\hline Lactation 1 & 252 & 5,575 & 9,825 \\
\hline Lactation 2 & 233 & 5,162 & 9,074 \\
\hline Lactation 3 & 121 & 709 & 1,028 \\
\hline Total & 271 & 11,446 & 19,927 \\
\hline \multicolumn{4}{|l|}{ Test } \\
\hline Lactation 1 & 245 & 1,623 & 3,748 \\
\hline Lactation 2 & 232 & 1,428 & 3,485 \\
\hline Lactation 3 & 91 & 372 & 767 \\
\hline Total & 271 & 3,423 & 8,000 \\
\hline Total & & 14,869 & 27,927 \\
\hline
\end{tabular}

${ }^{1}$ Lactation group 3 includes lactation number 3 and above.

to the Norwegian dairy goat control. The raw spectral data are also stored in a database for research and development purposes. For this study, 2 yr (2007 and 2008) of raw goat milk FTIR spectral data were used. Phenotype measurements (i.e., fat, protein, and lactose) were obtained from the Norwegian Dairy Goat Control recording system. These phenotypes were predictions based on the spectra. We use these predicted phenotypes because no phenotype was available from chemical analysis.

Two data sets were created randomly, one for calibrating a model (calibration data set) and other for testing model performance (test data set). The sizes of the data sets were 19,927 and 8,000 for calibration and test data sets, respectively. The structure of the data sets used in this study is presented in Table 1.

A pedigree file containing all animals with records was available. Their ancestors were traced back as far as possible and the final pedigree file contained 42,770 animals. The number of sires was 4,102 and number of dams was 20,644. Among the sires, 1,890 of them had progeny with records in the data set. Among the dams, 2,632 of them had both their own record and progeny with records, and 9,971 of them had progeny with records in the data set.

\section{Calibration of Prediction Model}

Due to the large amount of water in milk, both the $\mathrm{O}-\mathrm{H}$ bending region (between 1,600 and 1,700 $\mathrm{cm}^{-1}$ ) and the $\mathrm{O}-\mathrm{H}$ stretching region (above $3,020 \mathrm{~cm}^{-1}$ ) are more or less opaque to IR light in milk samples (Afseth et al., 2010). Another region, between 1,801 and 2,600 $\mathrm{cm}^{-1}$, does not contain useful chemical information (Andersen et al., 2002). These spectral regions were removed and the remaining frequencies (i.e., 941 to 1,586 $\mathrm{cm}^{-1}, 1,705$ to $1,801 \mathrm{~cm}^{-1}$, and 2,693 to $3,067 \mathrm{~cm}^{-1}$ ), giving a total of 321 spectral data points, were selected 
for calibration. The selected spectral variables were used to develop a multivariate calibration model based on partial least squares (PLS) regression (Martens and Næs, 1989). Partial least squares regression is widely used as a calibration method to establish calibration equations for prediction of milk composition traits based on spectroscopy. Three separate univariate PLS models were developed using fat, lactose, and protein percentages as response variables and the raw spectra as predictor variables for the calibration data set.

The models were developed using the pls package (Mevik and Wehrens, 2007) implemented in R (R Development Core Team, 2008). The prediction models were cross-validated using 10 random segments. All prediction residuals were then combined to compute the root mean square error of cross-validation (Hubert and Vanden Branden, 2003). The optimum number of PLS factors were determined by checking the change in root mean square error of cross-validation. The PLS regression models yielded regression coefficients $\left(\hat{\mathbf{B}}_{P L S_{i}}\right)$ for predicting fat, protein, and lactose percentages from FTIR spectra. Predicted breeding values (EBV) for fat, lactose, and protein percentages were obtained using 2 alternative prediction approaches.

\section{DP}

In this approach, the additive genetic variance components of all spectral variables and their breeding values need to be calculated first. Then, breeding values for any milk composition trait are predicted as a trait correlated to the genetic component of the FTIR spectra.

Direct estimation of genetic and other variance components for all spectral variables (i.e., 321 data points) simultaneously was not possible due to limitations of the current common quantitative genetic analysis methods (Meyer, 2007; Madsen and Jensen, 2008; Gilmour et al., 2009). Use of multivariate techniques such as principal components analysis (PCA) to reduce the spectral data dimension into fewer components (latent traits) was elaborated elsewhere (Soyeurt et al., 2010; Dagnachew and Ådnøy, 2011). A principal component decomposition of milk FTIR spectra in a vector form can be represented as follows:

$$
\mathbf{w}=\left(\mathbf{I}_{n} \otimes \mathbf{P}\right) \cdot \mathbf{t}+\varepsilon,
$$

where $\mathbf{w}$ is a vector of spectral variables (with the spectra of one sample above the other), $\mathbf{t}$ is a vector of scores (with the score of one sample on top of the other), $\mathbf{P}$ is a matrix of loadings, $\mathbf{I}_{n}$ is an identity matrix of size $n, n$ is the number of milk samples, $\otimes$ denotes the Kronecker product operator and $\varepsilon$ is a vector of residuals. The scores (t) are referred to as latent traits of the milk samples.

The following multitrait test-day animal model was assumed to find breeding values for the latent traits $\mathbf{t}$ :

$$
\mathbf{t}=\mathbf{X} \mathbf{b}_{\mathbf{t}}+\mathbf{Z} \mathbf{u}_{\mathbf{t}}+\mathbf{H h}_{\mathbf{t}}+\mathbf{e}_{\mathrm{t}}
$$

where $\mathbf{b}_{\mathbf{t}}$ is a vector of fixed effects (i.e., herd test day, kidding season, and year) for the latent traits, $\mathbf{u}_{\mathbf{t}}$ is a vector of breeding values for the latent traits, $\mathbf{h}_{\mathbf{t}}$ is a vector of permanent environmental effects, $\mathbf{e}_{t}$ is a vector of random residuals, and $\mathbf{X}, \mathbf{Z}$, and $\mathbf{H}$ are design matrices. The following (co)variance structure for the latent traits in $\mathbf{t}$ was assumed: $\operatorname{var}\left(\mathbf{u}_{\mathbf{t}}\right)=\mathbf{G}_{\mathbf{t}} \otimes \mathbf{A}$, $\operatorname{var}\left(\mathbf{h}_{\mathbf{t}}\right)=\mathbf{Q}_{\mathbf{t}} \otimes \mathbf{I}_{\mathbf{h}}$, and $\operatorname{var}\left(\mathbf{e}_{\mathbf{t}}\right)=\mathbf{R}_{\mathbf{t}} \otimes \mathbf{I}_{n}$, where $\mathbf{G}_{\mathbf{t}}$ is the genetic (co)variance of the latent traits, $\mathbf{A}$ is the numerator additive relationship matrix, $\mathbf{Q}_{\mathbf{t}}$ is the permanent environmental (co)variance, $\mathbf{R}_{\mathbf{t}}$ is the residual (co)variance, and $\mathbf{I}_{\mathbf{h}}$ and $\mathbf{I}_{n}$ are identity matrices of appropriate sizes.

With the information of the additive genetic relationship matrix $\mathbf{A}$ of individuals in the data set, REML estimates of the variance components of the latent traits $\widehat{\mathbf{G}_{\mathrm{t}}}, \widehat{\mathbf{Q}_{\mathrm{t}}}$, and $\widehat{\mathbf{R}_{\mathrm{t}}}$ were obtained using multivariate average information REML (AI-REML) algorithm of the WOMBAT program (Meyer, 2007).

Equation 1.2 yields $\widetilde{\mathbf{u}_{\mathbf{t}}}$, a vector of predicted breeding values for the latent traits by use of the mixed-model equation. Through use of linear transformation of Equation 1.1, breeding values of the latent traits were back transformed to the spectral variable breeding values using a transformation equation:

$$
\widetilde{\mathbf{u}_{w}}=\left(\mathbf{I}_{n} \otimes \mathbf{P}\right) \cdot \widetilde{\mathbf{u}_{\mathbf{t}}}
$$

where $\widetilde{\mathbf{u}_{w}}$ are predicted breeding values for the spectral variables (genetic components of FTIR spectra). Similarly, permanent $\left(\widetilde{\mathbf{h}_{w}}\right)$ and temporary $\left(\widetilde{\mathbf{e}_{w}}\right)$ environmental components of the FTIR spectra were found by replacing respective values in the transformation equation i.e., $\widetilde{\mathbf{h}_{w}}=\left(\mathbf{I}_{n} \otimes \mathbf{P}\right) \cdot \widetilde{\mathbf{h}_{\mathbf{t}}}$ and $\left.\widetilde{\mathbf{e}_{w}}=\left(\mathbf{I}_{n} \otimes \mathbf{P}\right) \cdot \widetilde{\mathbf{e}_{\mathbf{t}}}\right]$.

Breeding values for a milk component trait, $\mathbf{y}_{i}(i=1$, 2 , or 3 ; i.e., fat, lactose, and protein percentages), were then predicted as correlated traits from the genetic component of FTIR spectra $\left(\widetilde{\mathbf{u}_{w}}\right)$.

$$
\tilde{\mathbf{u}}_{i}^{*}=\left(\mathbf{I}_{n} \otimes \hat{\mathbf{B}}_{P L S_{i}}{ }^{\prime}\right) \cdot \widetilde{\mathbf{u}_{w}}=\left(\mathbf{I}_{n} \otimes \hat{\mathbf{B}}_{P L S_{i}}{ }^{\prime}\right) \cdot\left[\left(\mathbf{I}_{n} \otimes \mathbf{P}\right) \cdot \widetilde{\mathbf{u}_{\mathbf{t}}}\right],
$$

where $\tilde{\mathbf{u}}_{i}^{*}$ is a vector of predicted breeding values for a trait $\mathbf{y}_{i}$ under the DP approach. 
Table 2. Summary of partial least squares (PLS) regression prediction models for fat, lactose, and protein percentages $^{1}$

\begin{tabular}{lcccc}
\hline Trait & $\begin{array}{c}\text { Samples } \\
\text { (no.) }\end{array}$ & $\begin{array}{c}\text { PLS factors } \\
\text { (no.) }\end{array}$ & RMSECV & R $_{\text {cv }}{ }^{2}$ \\
\hline Fat percentage & 19,927 & 8 & 0.1856 & 0.97 \\
Lactose percentage & 19,927 & 10 & 0.0616 & 0.95 \\
Protein percentage & 19,927 & 8 & 0.0437 & 0.98 \\
\hline
\end{tabular}

${ }^{1} \mathrm{RMSECV}=$ root mean square error of cross-validation; $\mathrm{R}_{\mathrm{cv}}{ }^{2}=$ coefficient of determination from crossvalidation.

\section{IP}

This is the common approach used today for genetic evaluation of dairy animals. In this approach, first milk composition phenotypes are predicted from the FTIR spectra and then genetic parameters and breeding values are estimated using the predicted phenotypes.

Prediction models were calibrated using PLS regression as described earlier. Phenotypes for a milk composition trait were then predicted from the spectra using the PLS regression coefficients:

$$
\hat{\mathbf{y}}_{i}=\left(\mathbf{I}_{\mathrm{n}} \otimes \hat{\mathrm{B}}_{\mathrm{PLS}_{\mathrm{i}}}{ }^{\prime}\right) \cdot \mathbf{w} \quad(\mathrm{i}=1,2, \text { or } 3),
$$

where $\mathbf{w}$ is as defined in Equation 1.1, $\hat{\mathbf{y}}_{i}$ is the predicted phenotype for milk composition trait $i(i=1,2$, or 3$)$, and $\hat{B}_{P L S_{i}}$ are PLS regression coefficients for trait $i$. We assume similar effects as in Equation 1.2, but a single-trait test-day animal model for the predicted phenotype as follows:

$$
\hat{\mathbf{y}}_{\mathrm{i}}=\mathbf{X}_{i} \mathbf{b}_{i}+\mathbf{Z}_{i} \mathbf{u}_{i}+\mathbf{H}_{i} \mathbf{h}_{i}+\mathbf{e}_{i} .
$$

The model components were as defined in Equation 1.2 , but with a univariate (co)variance structure: $\operatorname{var}\left(\mathbf{u}_{i}\right)=\mathbf{A} \cdot \sigma_{a_{i}}^{2}, \operatorname{var}\left(\mathbf{h}_{i}\right)=\mathbf{I}_{\mathbf{h}} \cdot \sigma_{p e_{i}}^{2}$, and $\operatorname{var}\left(\mathbf{e}_{i}\right)=\mathbf{I}_{n}$. $\sigma_{e_{i}}^{2}$, where $\sigma_{a_{i}}^{2}$ is the additive genetic variance of trait $i$, $\sigma_{p e_{i}}^{2}$ is its permanent environmental variance, and $\sigma_{e_{i}}^{2}$ is the residual variance. For a given data set $\hat{\mathbf{y}}_{i}$ of $n$ observations of a trait and information on additive genetic relationship A of individuals in the data set, it is possible to find REML estimates of the variance components of the predicted phenotypes. However, to compare the two DP and IP methods under equal (co)variance structures, the variance components for single traits were taken from the (co)variances of the spectra:

$$
\sigma_{a_{i}}^{2}=\hat{B}_{P L S_{i}}{ }^{\prime} \cdot \mathbf{P} \cdot \widehat{\mathbf{G}_{\mathbf{t}}} \cdot \mathbf{P}^{\prime} \cdot \hat{B}_{P L S_{i}} .
$$

In a similar manner, the permanent environmental $\left(\sigma_{p e_{i}}^{2}\right)$ and residual $\left(\sigma_{e_{i}}^{2}\right)$ variances were found using Equation 2.3 by replacing the respective values (i.e., $\sigma_{p e_{i}}^{2}=\hat{B}_{P L S_{i}}{ }^{\prime} \cdot \mathbf{P} \cdot \widehat{\mathbf{Q}_{\mathbf{t}}} \cdot \mathbf{P}^{\prime} \cdot \hat{B}_{P L S_{i}}$ and $\left.\sigma_{e_{i}}^{2}=\hat{B}_{P L S_{i}}{ }^{\prime} \cdot \mathbf{P} \cdot \widehat{\mathbf{R}_{\mathbf{t}}} \cdot \mathbf{P}^{\prime} \cdot \hat{B}_{P L S_{i}}\right)$. Application of the mixed-model equation to Equation 2.2 yields $\hat{\mathbf{u}}_{i}$, a vector of breeding values of trait $y_{i}$ under the IP approach.

So, breeding values for any milk composition trait $y_{i}$ can be found by 2 approaches: $\hat{\mathbf{u}}_{i}$ (using IP) or $\tilde{\mathbf{u}}_{i}^{*}$ (using DP). Therefore, the question is which BLUP value gives better genetic gain for the trait $\hat{\mathbf{y}}_{i}$. Diagrammatical representations of the DP and IP methods are presented in Figure 1. The figure demonstrates that for the IP approach, the genetic analysis (REML and BLUP) is done for as many traits as needed separately, whereas in the DP approach, this is done once.

\section{Model Evaluation}

Performances of the 2 approaches for prediction of breeding values were tested on a separate data set (validation; Table 1 ). The 2 vectors of breeding values $\left(\hat{\mathbf{u}}_{i}\right.$ and $\left.\tilde{\mathbf{u}}_{i}^{*}\right)$ are 2 different predictions of the same breeding values, each with their associated PEV. Prediction error variances were obtained from the inverse of the coefficient matrix of the respective mixed models. Given the prediction error variances, mean accuracies and relative genetic gains were also determined for both approaches following the definition of accuracy in mixed models (Lynch and Walsh, 1998). Predicted breeding values were also compared using the Spearman rank correlation coefficient of R ( $\mathrm{R}$ Development Core Team, 2008).

\section{RESULTS AND DISCUSSION}

\section{Multivariate Calibration of Prediction Models}

Regression models were developed for predicting fat, lactose, and protein percentages from FTIR spectra using PLS models. Table 2 presents a summary of the prediction models developed for these traits. The coefficient of determination from cross-validation $\left(\mathrm{R}_{\mathrm{cv}}{ }^{2}\right)$ varied from 0.98 for protein to 0.95 for lactose (Table 2). These models are considered as good prediction models (De Marchi et al., 2009). The observed $\mathrm{R}_{\mathrm{cv}}{ }^{2}$ values are relatively higher than reported in the literature for non- 
commercial calibration (Etzion et al., 2004; Bonfatti et al., 2011). A reason for these higher coefficients of determination might be that the phenotypes used for calibrating the models were obtained from previous prediction based on the same FTIR spectra.

\section{FTIR Spectra Genetic Component}

The genetic component of the FTIR spectra was estimated after reducing the spectral dimension using PCA. The PCA yielded 8 components (latent traits) explaining $99.05 \%$ of the total spectral variation. Table
3 presents relative variance explained by the latent traits and, REML estimates of genetic, permanent environmental, and random residual variance ratios of the latent traits. The genetic variance ratios (heritability) of the latent traits varied from 0.198 to 0.382 . The permanent environmental effect ratios were between 0.135 and 0.227 and the residual variance ratios ranged from 0.404 to 0.634 (Table 3). The magnitudes of genetic correlation among the latent traits ranged from 0.013 to 0.512 and the magnitudes of the residual correlation among the latent traits were between 0.001 and 0.449 (results not presented).

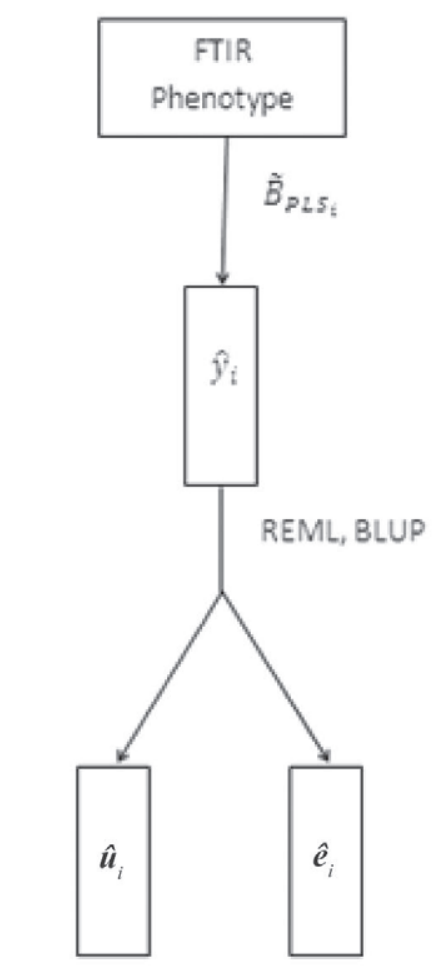

Indirect prediction (IP)

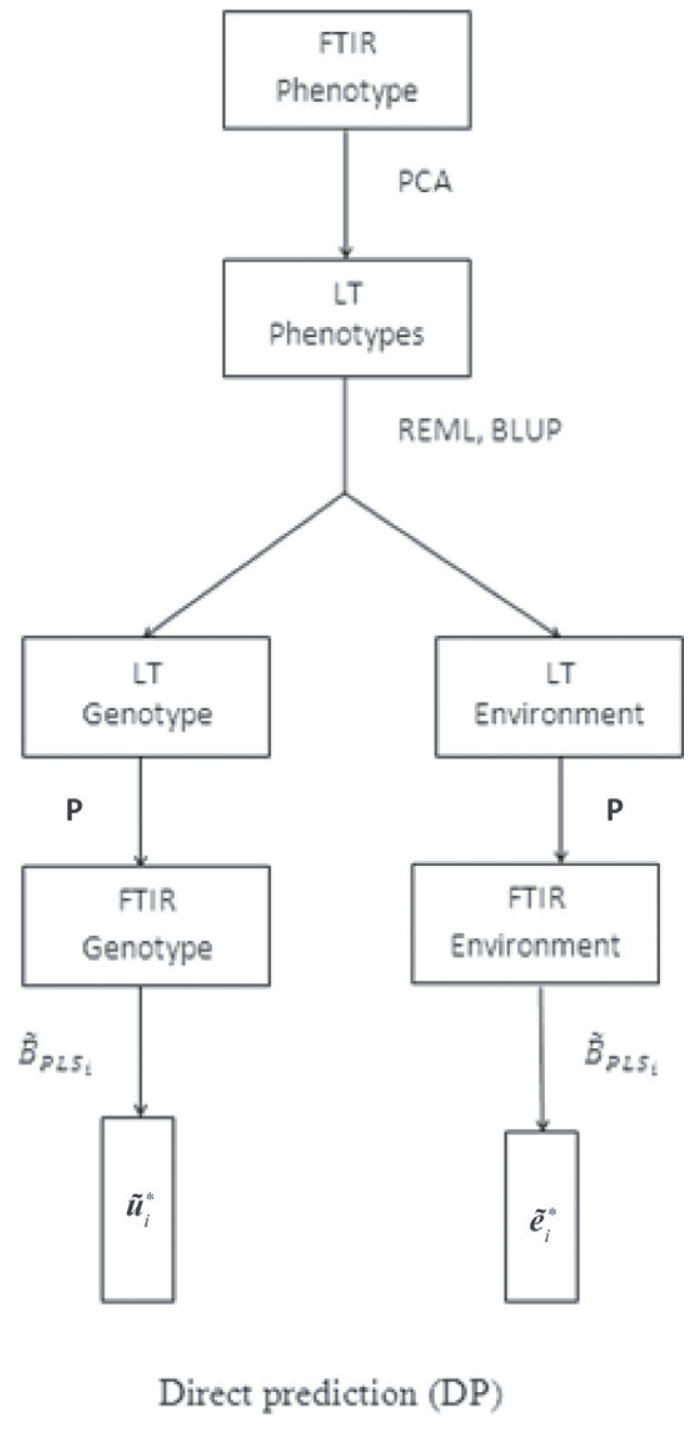

Figure 1. Schematic representation of the indirect (IP) and direct (DP) prediction approaches. Whereas for IP the genetic analysis (REML and BLUP) is done for as many traits as needed separately, in the DP this is done once. FTIR $=$ Fourier-transform infrared; $\hat{B}_{P L S_{i}}=$ regression coefficient for trait $i ; \hat{\mathbf{y}}_{i}=$ predicted phenotype for milk composition trait $i(i=1,2$, or 3$)$; $\hat{\mathbf{u}}_{i}=$ a vector of breeding values of trait $y_{i}$ under the IP approach; $\hat{\mathbf{e}}_{i}=$ a vector of random residual under the IP approach; LT $=$ latent traits; PCA $=$ principal components analysis; $\mathbf{P}=$ a matrix of loadings; $\tilde{\mathbf{u}}_{i}^{*}=$ a vector of predicted breeding values for a trait $y_{i}$ under the DP approach; $\tilde{\mathbf{e}}_{i}^{*}=$ a vector of random residual under the DP approach. 
Table 3. Estimates $( \pm \mathrm{SE})$ of variance ratios for genetic, permanent environmental, and residual random effects

\begin{tabular}{lcccc}
\hline & & \multicolumn{3}{c}{ Variance ratio $^{1}$} \\
\cline { 3 - 4 } & $\begin{array}{c}\text { Variance } \\
\text { Item }\end{array}$ & Permanent \\
explained (\%) & Genetic & & Residual \\
\hline Latent trait & & & & \\
1 & 49.63 & $0.253 \pm 0.020$ & $0.135 \pm 0.021$ & $0.613 \pm 0.013$ \\
2 & 35.74 & $0.382 \pm 0.022$ & $0.214 \pm 0.022$ & $0.404 \pm 0.009$ \\
3 & 5.95 & $0.330 \pm 0.021$ & $0.199 \pm 0.021$ & $0.471 \pm 0.010$ \\
4 & 4.74 & $0.257 \pm 0.020$ & $0.227 \pm 0.021$ & $0.516 \pm 0.011$ \\
5 & 1.63 & $0.371 \pm 0.021$ & $0.197 \pm 0.022$ & $0.432 \pm 0.010$ \\
6 & 0.56 & $0.303 \pm 0.021$ & $0.163 \pm 0.021$ & $0.533 \pm 0.011$ \\
7 & 0.47 & $0.198 \pm 0.019$ & $0.192 \pm 0.021$ & $0.610 \pm 0.012$ \\
8 & 0.33 & $0.205 \pm 0.019$ & $0.161 \pm 0.020$ & $0.634 \pm 0.012$ \\
Total variance & 99.05 & & & \\
explained & & & &
\end{tabular}

${ }^{1}$ The ratio is the relative to total phenotypic variance for each latent trait.

Studies on bovine milk spectra (Soyeurt et al., 2010) and goat milk spectra (Dagnachew and Ådnøy, 2011) show that spectral variables are highly correlated with each other. The results of the PCA in the current study are in support of those studies. Table 3 shows that only 8 components (latent traits) were enough to explain approximately $99 \%$ of the total spectral variation, indicating strong correlation among the 321 spectral variables.

The estimated (co)variance structure of the FTIR spectra are used to estimate the variance components of the milk composition traits (i.e., fat, lactose, and protein) using Equation 2.3. Table 4 presents estimated heritability, genetic, permanent environmental, and residual variance components of fat, lactose, and protein content of the milk. The estimated heritabilities were $0.258,0.322$, and 0.315 for fat, lactose, and protein, respectively. These results are slightly smaller than values estimated by the genetic evaluation program of Norwegian dairy goats. Fat, lactose, and protein heritabilities of $0.271,0.376$, and 0.356 are used in the Norwegian goat breeding scheme [I. A. Boman, Norwegian Association of Sheep and Goat Breeders (NSG), Ås, Norway, personal communication, 2009). However, those heritabilities were estimated using larger data sets.

\section{PEV}

One approach to measure the approximate relative gain due to the use of the DP instead of the IP approach for prediction of breeding values is through comparing PEV (Schaeffer, 1984). Table 5 presents a summary of EBV under the 2 approaches, mean PEV, and relative reductions in mean PEV. The table shows that the use of the DP approach reduced the mean PEV by $3.73 \%$ for fat, $4.07 \%$ for lactose, and $7.06 \%$ for protein.

The main difference between the IP and DP approaches is that the genetic analysis is carried out on the spectral variables for the latter method. Thus, DP uses more information to evaluate animals through genetic and environmental correlations among the spectral variables (Thompson and Meyer, 1986). The DP method could be thought of as a multitrait analysis on several hundreds of spectral traits (i.e., 321 traits in the current study). Therefore, the observed reductions in mean PEV (Table 5) were due to the fact that it combined the genetic relationship between all spectral traits. These results are in agreement with studies testing the power of multitrait over single-trait models (Schaeffer, 1984; Gengler and Coenraets, 1996). Gengler and Coenraets (1996) reported a reduction in PEV up to $6 \%$ for fat yield and $0.4 \%$ for protein yield by

Table 4. Estimated heritability ${ }^{1}$ and genetic $\left(\sigma_{a}^{2}\right)$, permanent environmental $\left(\sigma_{p e}^{2}\right)$, and random residual $\left(\sigma_{e}^{2}\right)$ variance components for fat, lactose, and protein percentages

\begin{tabular}{lcccc}
\hline Trait & $\mathrm{h}^{2}$ & $\sigma_{a}^{2}$ & $\sigma_{p e}^{2}$ & $\sigma_{e}^{2}$ \\
\hline Fat percentage & 0.258 & 0.1454 & 0.0779 & 0.3384 \\
Lactose percentage & 0.322 & 0.0109 & 0.0087 & 0.0144 \\
Protein percentage & 0.315 & 0.0135 & 0.0101 & 0.0193 \\
\hline${ }^{1}$ Where ${ }^{2}=\frac{\sigma_{a}^{2}}{\sigma_{a}^{2}+\sigma_{p e}^{2}+\sigma_{e}^{2}}$. & & &
\end{tabular}




\section{General Discussion}

Implementation of breeding schemes that aim at improving milk quality require identification of superior individuals and keeping them for breeding. In this study, genetic evaluation of animals using the DP approach and the IP approach were compared for fat, protein, and lactose percentages. The results show that the DP method improved the accuracy of identifying best individuals for all traits tested in the study. But lowly heritable milk quality traits could benefit more from the proposed method because it evaluates animals through genetic correlations of many spectral variables.

The effectiveness of FTIR spectra for the prediction of milk coagulation properties and detailed protein composition has been investigated in bovine milk (Hewavitharana and van Brakel, 1997; Dal Zotto et al., 2008; De Marchi et al., 2009; Bonfatti et al., 2011; Rutten et al., 2011). Even though the ability of FTIR spectroscopy to predict milk coagulation properties and protein composition is not satisfactory, the predictions could be used as indicator traits in selective breeding to enhance these properties (Cecchinato et al., 2009; Bonfatti et al., 2011; Rutten et al., 2011). Further improving nutritional quality of dairy products through inclusion of the FA composition profile in animal selection programs is possible because mid-infrared spectroscopy is able to predict the milk FA profile (Soyeurt et al., 2006; Afseth et al., 2010). To this end, with all this information being available for breeding, following the IP approach may require acquisition of several phenotypic traits and multiple genetic analyses. However, in the DP approach, for traits to be predicted from spectra, no need exists to estimate variance components several times, as these can be calculated from the variance components of the spectra. Breeding values of all traits could be found directly from the genetic component of the FTIR spectra, and more accurately with DP than with IP. Today, several milk traits are predicted form FTIR spectra and calibration equations (i.e., regression models) are available for many traits. The DP approach could take advantage through using already established regression coefficients to predict breeding values for these milk traits from the genetic components of the spectra.

The application of the DP approach is not limited to milk composition traits obtained from milk IR spectroscopy, but could also be extended to any trait predicted from any spectroscopy method. Genetic evaluation in many species use spectroscopy techniques for acquisition of phenotypes, for instance, in beef cattle breeding (e.g., Cecchinato et al., 2011a), pig breeding (e.g., Gjerlaug-Enger, 2011), and fish breeding (e.g., Gjerde and Martens, 1987). The principles of the DP approach could also be implemented in these breeding programs for estimation of variance components and prediction of breeding values.

The variance components of individual milk composition traits in the IP approach were here calculated from the variance components of the FTIR spectra (using Equation 2.3) for the purpose of comparing the two models with the same (co)variance structure. This might reduce the difference in performance between the two approaches (in favor of IP) because DP used information from correlated spectral traits to evaluate animals and is, thus, likely to yield more accurate variance component estimates (Schaeffer, 1984; Meyer, 1991). Further investigation of the approaches using phenotypic data obtained by chemical analysis is required to determine the power of the DP approach.

In this study, PCA was used to reduce the spectral dimension because it was not possible to estimate variance components for all spectral variables (i.e., 321 spectral data points) simultaneously. This dimensionreduction step could also be achieved using PLS analysis for the intended milk composition traits. This might yield a more accurate estimation of genetic parameters for the traits included in the PLS model because it tends to direct the latent variables to capture relevant variations (information) for the intended traits. However, it will not guarantee that information for other milk composition traits, which are not included in the PLS model, are retained in the latent variables. On the other hand, the PCA model extracts information, unselectively focusing only on the magnitude of total variation explained by a component. In the current study, the 8 latent traits (components) explaining approximately $99 \%$ of the total spectral variation were used in extracting the genetic component of the FTIR spectra. The remaining $1 \%$ of the total spectral variation could also have relevant information for breeding. Soyeurt et al. (2010) integrated PCA with canonical transformation and used more latent trait (46 components) in extracting the genetic component of spectral data. The method can be implemented to capture part of the remaining $1 \%$ of the spectral variation.

\section{CONCLUSIONS}

Genetic evaluation of individuals based on several hundred milk FTIR spectral variables - DP approachyielded more accurate EBV for fat, lactose, and protein content of milk compared with first predicting phenotypes and then genetic evaluation of FTIR-predicted traits - IP approach. Moreover, lowly heritable traits could benefit more from the proposed approach, as it uses information from strongly correlated spectral traits to evaluate animals. To this end, with more de- 
tailed milk composition information from FTIR spectra becoming available for breeding, the DP approach has strong potential in dairy animal breeding schemes. Furthermore, the potential and application of the DP method is not only limited to milk FTIR spectra, but could also be extended to any spectroscopy techniques implemented in other species and for other traits.

\section{ACKNOWLEDGMENTS}

This study was financially supported by the Research Council of Norway (Oslo, Norway), the Norwegian Dairy Association (TINE; Oslo, Norway), and the Norwegian University of Life Sciences (UMB; Ås, Norway). The authors gratefully acknowledge TINE for providing the milk FTIR spectral data.

\section{REFERENCES}

Afseth, N. K., H. Martens, A. Randby, L. Gidskehaug, B. Narum, K. Jørgensen, S. Lien, and A. Kohler. 2010. Predicting the fatty acid composition of milk: A comparison of two Fourier transform infrared sampling techniques. Appl. Spectrosc. 64:700-707.

Andersen, S. K., P. W. Hansen, and H. V. Andersen. 2002. Vibrational spectroscopy in the analysis of dairy products and wine. Pages 3672-3681 in Handbook of Vibrational Spectroscopy. J. M. Chalmers and P. R. Griffiths, ed. John Wiley \& Sons Ltd., West Sussex, UK.

Bonfatti, V., G. Di Martino, and P. Carnier. 2011. Effectiveness of mid-infrared spectroscopy for the prediction of detailed protein composition and contents of protein genetic variants of individual milk of Simmental cows. J. Dairy Sci. 94:5776-5785.

Cassandro, M., A. Comin, M. Ojala, R. D. Zotto, M. De Marchi, L. Gallo, P. Carnier, and G. Bittante. 2008. Genetic parameters of milk coagulation properties and their relationships with milk yield and quality traits in Italian Holstein cows. J. Dairy Sci. 91:371-376.

Cecchinato, A., M. De Marchi, L. Gallo, G. Bittante, and P. Carnier. 2009. Mid-infrared spectroscopy predictions as indicator traits in breeding programs for enhanced coagulation properties of milk. J. Dairy Sci. 92:5304-5313.

Cecchinato, A., M. De Marchi, M. Penasa, A. Albera, and G. Bittante. 2011a. Near-infrared reflectance spectroscopy predictions as indicator traits in breeding programs for enhanced beef quality. J. Anim. Sci. 89:2687-2695.

Cecchinato, A., M. Penasa, M. De Marchi, L. Gallo, G. Bittante, and P. Carnier. 2011b. Genetic parameters of coagulation properties, milk yield, quality, and acidity estimated using coagulating and noncoagulating milk information in Brown Swiss and HolsteinFriesian cows. J. Dairy Sci. 94:4205-4213.

Dagnachew, B. S., and T. Ådnøy. 2011. Genetic and environmental variability of goat milk FTIR spectra. Page 110 in Book of Abstracts of the 62nd Annual Meeting of the European Federation of Animal Science. Wageningen Academic Publishers, Wageningen, the Netherlands.

Dal Zotto, R., M. De Marchi, A. Cecchinato, M. Penasa, M. Cassandro, P. Carnier, L. Gallo, and G. Bittante. 2008. Reproducibility and repeatability of measures of milk coagulation properties and predictive ability of mid-infrared reflectance spectroscopy. J. Dairy Sci. 91:4103-4112.

De Marchi, M., C. C. Fagan, C. P. O'Donnell, A. Cecchinato, R. Dal Zotto, M. Cassandro, M. Penasa, and G. Bittante. 2009. Prediction of coagulation properties, titratable acidity, and $\mathrm{pH}$ of bovine milk using mid-infrared spectroscopy. J. Dairy Sci. 92:423-432.
Etzion, Y., R. Linker, U. Cogan, and I. Shmulevich. 2004. Determination of protein concentration in raw milk by mid-infrared Fourier transform infrared/attenuated total reflectance spectroscopy. J. Dairy Sci. 87:2779-2788.

Falconer, D. S. 1989. Introduction to Quantitative Genetics. 3rd ed. Longman Scientific and Technical Harlow, UK.

Gengler, N., and P. Coenraets. 1996. Use of multiple-trait animal models for genetic evaluation of milk, fat and protein lactation yields of dairy in Belgium. Biotechnol. Agron. Soc. Environ. 1:26-33.

Gilmour, A. R., B. J. Gogel, B. R. Cullis, and R. Thompson. 2009 ASReml User Guide. Release 3.0. VSN International Ltd., Hemel Hempstead, UK.

Gjerde, B., and H. Martens. 1987. Predicting carcass composition of rainbow trout by near-infrared reflectance spectroscopy. J. Anim. Breed. Genet. 104:137-148.

Gjerlaug-Enger, E. 2011. Genetic analysis of meat, fat and carcass quality traits measured by rapid methods. PhD Thesis. Department of Animal and Aquacultural Sciences, Norwegian University of Life Sciences, Ås, Norway.

Goulden, J. D. S. 1964. Analysis of milk by infra-red absorption. J. Dairy Res. 31:273-284. http://dx.doi.org/10.1017/ S0022029900018203.

Grosclaude, F., and P. Martin. 1997. Casein polymorphisms in the goat. Pages 241-253 in Proc. International Dairy Federation, Palmerston North, New Zealand.

Hallén, E. 2008. Coagulation properties of milk: Association with milk protein composition and genetic polymorphism. Doctoral Thesis. Faculty of Natural Resources and Agricultural Sciences, Department of Food Science, Swedish University of Agricultural Sciences, Uppsala, Sweden.

Henderson, C. R. 1975. Best linear unbiased estimation and prediction under a selection model. Biometrics 31:423-447.

Hewavitharana, A. K., and B. van Brakel. 1997. Fourier transform infrared spectrometric method for the rapid determination of casein in raw milk. Analyst (Lond.) 122:701-704

Hubert, M., and K. Vanden Branden. 2003. Robust methods for partial least squares regression. J. Chemometr. 17:537-549.

Ikonen, T. 2000. Possibilities of genetic improvement of milk coagulation properties of dairy cows. PhD Diss. Faculty of Agriculture and Forestry, University of Helsinki, Helsinki, Finland.

Jaubert, G. 1996. Biochemical characteristics and quality of goat milk. Pages 71-74 in International Conference on Goats. P. MorandFehr, ed. CIHEAM, Beijing, China.

Lynch, M., and B. Walsh. 1998. Genetics and Analysis of Quantitative Traits. Sinauer Associates Inc., Sunderland, MA.

Madsen, P., and J. Jensen. 2008. An User's Guide to DMU. A package for analyzing multivariate mixed models. Version 6 , release 4.7. University of Aarhus, Faculty of Agricultural Sciences (DFJ), Dept. Genetics and Biotechnology, Research Centre Foulum, Tjele, Denmark.

Manfredi, E. 2003. The tale of goat alpha-s1 casein. Proc. International Workshop on Major Genes and QTL in Sheep and Goat. CD-ROM Communication No. 2-31. INRA, Toulouse, France.

Martens, H., and T. Næs. 1989. Multivariate Calibration. John Wiley \& Sons Ltd., Chichester, UK.

Mevik, B.-H., and R. Wehrens. 2007. The pls package: Principal component and partial least squares regression in R. J. Stat. Softw. $18: 1-24$.

Meyer, K. 1991. Estimating variances and covariances for multivariate animal models by restricted maximum likelihood. Genet. Select. Evol. 23:67-83.

Meyer, K. 2007. WOMBAT-A tool for mixed model analyses in quantitative genetics by restricted maximum likelihood (REML). J. Zhejiang Univ. Sci. B 8:815-821.

Newcom, D. W., T. J. Baas, K. J. Stalder, and C. R. Schwab. 2005. Comparison of three models to estimate breeding values for percentage of loin intramuscular fat in Duroc swine. J. Anim. Sci. 83:750-756.

R Development Core Team. 2008. R: A language and environment for statistical computing. R Foundation for Statistical Computing, Vienna, Austria. http://www.R-project.org. 
Rutten, M. J. M., H. Bovenhuis, J. M. L. Heck, and J. A. M. van Arendonk. 2011. Predicting bovine milk protein composition based on Fourier transform infrared spectra. J. Dairy Sci. 94:5683-5690.

Schaeffer, L. R. 1984. Sire and cow evaluation under multiple trait models. J. Dairy Sci. 67:1567-1580.

Schopen, G. C. B., J. M. L. Heck, H. Bovenhuis, M. H. P. W. Visker, H. J. F. van Valenberg, and J. A. M. van Arendonk. 2009. Genetic parameters for major milk proteins in Dutch Holstein-Friesians. J. Dairy Sci. 92:1182-1191.

Sigurdsson, A., and T. Arnason. 1995. Predicting genetic trend by uniand multitrait models. Acta Agric. Scand. A Anim. Sci. 45:1-10.

Soyeurt, H., P. Dardenne, F. Dehareng, G. Lognay, D. Veselko, M. Marlier, C. Bertozzi, P. Mayeres, and N. Gengler. 2006. Estimating fatty acid content in cow milk using mid- infrared spectrometry. J. Dairy Sci. 89:3690-3695.

Soyeurt, H., I. Misztal, and N. Gengler. 2010. Genetic variability of milk components based on mid-infrared spectral data. J. Dairy Sci. 93:1722-1728.

Thompson, R., and K. Meyer. 1986. A review of theoretical aspects in the estimation of breeding values for multi-trait selection. Livest. Prod. Sci. 15:299-313.

van de Voort, F. R. 1992. Fourier transform infrared spectroscopy applied to food analysis. Food Res. Int. 25:397-403. 